Research Article

\title{
Intelligent Environment Platform for Industrial Clusters Based on Cloud Computing Technology
}

\author{
Wensheng Dai (i) \\ Financial School, China Financial Policy Research Center, International Monetary Institute, Renmin University of China, \\ Beijing 100872, China \\ Correspondence should be addressed to Wensheng Dai; daiws@ruc.edu.cn
}

Received 15 August 2021; Revised 15 October 2021; Accepted 21 October 2021; Published 1 November 2021

Academic Editor: Sang-Bing Tsai

Copyright (c) 2021 Wensheng Dai. This is an open access article distributed under the Creative Commons Attribution License, which permits unrestricted use, distribution, and reproduction in any medium, provided the original work is properly cited.

With the development of science and technology and the improvement of industrialization, the development of more and more industries has interacted to form many industrial clusters. For the healthy development and safety of various industries, environmental quality monitoring and management in industrial clusters is of utmost importance. Currently, domestic and foreign enterprises and related departments are vigorously developing smart environment platforms. The purpose of this paper is to design an intelligent environment platform for industrial clusters based on cloud computing technology. This article first uses the methods of literature research and network investigation to collect relevant literature and research results and organizes statistics on the collected information. Then, through case analysis to study the needs and overview of the construction of smart environment platforms in industrial clusters, it uses the wireless smart sensing technology of the Internet of Things and the information interaction technology of the Internet to provide data to cloud computing through mobile terminals, and cloud computing provides various types on demand data service. Then, according to the demand analysis of the intelligent environment platform, the functional modules and operation procedures of the intelligent environment platform are designed. The main monitoring content is water quality testing, air quality testing, garbage disposal and soil quality testing, etc. Finally, this article processes, analyzes, and predicts the detected sampled data through cloud computing. It can also locate and track abnormal data. Through the curve fitting of the data, the environmental conditions in the area can be predicted. Experiments show that the prediction accuracy rate is as high as $93.8 \%$, which plays an important role in monitoring and preventing environmental pollution.

\section{Introduction}

1.1. Background and Significance. With the development of various industries, especially the improvement of industrial production levels, a large number of interactions and integrations have occurred between various industries, forming many industrial clusters. In the past, environmental pollution problems were mainly aimed at the pollution of air and water quality caused by waste gas, heat, sewage, and dust particles generated in the production workshop during industrial production. However, with the formation of industrial clusters, cross-contamination has occurred in many areas. This is caused by the different accumulation of waste generated by different industries. The waste discharge treatment of some industrial manufacturers meets the standards, but the same as other industries. It caused environmental pollution and endangered people's health and industrial development. Therefore, people pay more and more attention to the environmental monitoring of industrial clusters while paying attention to the industrial production environment [1]. The main purpose of this research is to analyze and predict the environment under the information interaction based on intelligent sensor technology. In fact, the environmental pollution of industrial clusters may be more terrible than a single industrial pollution. The accumulation of various industrial wastes has caused unknown changes in the factors and composition of these environmental pollution and brought new difficulties to environmental governance. Some pollutants can be perceived by human senses, 
while the pollution of some factors such as air, water quality, and heavy metals is difficult to observe intuitively. The rapid development of information technology and the arrival of the cloud era have brought new opportunities to effectively solve these environmental problems. Through the wireless sensor technology of the Internet of Things, combined with various smart sensors, the environmental data in the industrial cluster can be monitored and updated in real time [2]. The technological development of sensors can be divided into three eras as a whole; the first generation uses structural parameter changes to experience and the structure type that converts the signal, the second generation is a solid type made of certain characteristics of solid component materials such as semiconductors, dielectrics, and magnetic materials, and the third generation is the so-called smart sensor. The data can be processed, analyzed, and predicted by uploading to the cloud server through the Internet connection. It can effectively monitor and prevent the occurrence of environmental pollution in industrial clusters. With the development of social networks, pictures have become the main carrier of information, which affects the efficiency of information retrieval. In this case, picture recognition technology is particularly important. With the development of the times, this technology has also started from its infancy; it has been used as a tool to meet entertainment needs. It has entered an advanced stage; artificial intelligence-based machines with vision can better perform their functions in information retrieval.

1.2. Related Research at Home and Abroad. In recent years, the environmental pollution caused by domestic industrial development has become increasingly serious, and relevant departments have paid more and more attention to the environmental monitoring and management of various industrial clusters. Yang et al. proposed to build an intelligent indoor environment monitoring system combined with ZigBee wireless sensor network technology and use information technology based on big data and cloud computing environment to develop a monitoring system to issue warnings and monitor the indoors in public areas, such as schools, offices, homes, or other buildings. Air quality ensures better air quality for people in the building [3]. Based on cloud computing technology, Zhang et al. provide computers and other equipment with shared computer processing and storage resources on demand and have the ability to share programs, data, and software, build a distributed collaborative urban traffic big data system, and promote people in different geographic locations to solve the problem of traffic search [4]. Based on the related concepts of Industry 4.0, Wan builds an intelligent manufacturing environment platform based on mobile services and cloud computing technology to meet the needs of custom manufacturing in the industry [5]. Zhao et al. designed an integrated data acquisition system based on cloud computing technology for intelligent monitoring equipment in the industrial field. At present, the equipment will be applied to Baosteel's acid regeneration environment monitoring device for data acquisition [6].
In foreign countries, the development of industrialization was much earlier than that in China. Therefore, as early as the 18th century, Western countries encountered environmental pollution problems caused by industrialization. In response to this, Western countries have also adopted many environmental monitoring and management protection measures. Ahmed et al. propose a robotized wireless sensor network that is suitable for solving different environmental monitoring tasks. Such robots can autonomously move to find and monitor polluted areas, helping to improve the efficiency of existing conventional pollution prevention systems, that is, to integrate existing wireless sensors The network is transformed into an adaptive sensor system with intelligent behavior, which can be used to find and track the location of polluted areas [7]. Scilimati et al. analyzed the IoT robot system used for environmental monitoring through experiments and found that the drone will not incur any significant overhead due to the onboard IoT device. The overall service quality represented by the data retrieval delay and packet loss rate meets the mission requirements. Robot systems can reveal potentially destructive potential [8]. Modern networks involve not only computers, but all kinds of other interconnected devices, from mobile phones to other household items equipped with sensors. In response to the inherent difficulties of modeling problems caused by these visions of the Internet of Things, Laghari and Niazi explore the use of agent-based modeling methods as part of a cognitive agent-based computing framework to model complex communication network problems. This modeling method has been applied to an autonomous multi-agent architecture that manages the carbon footprint of a company's network. Cloud computing also has many applications in other fields [9]. For example, Pareek and Patidar proposed a grayscale medical image encryption method based on the characteristics of genetic algorithms based on the characteristics of cloud computing. Performance analysis shows that the scheme has good statistical performance, key sensitivity, and can effectively resist brute force attacks, differential attacks, plaintext attacks, and entropy attacks [10]. In order to effectively solve the problem of inconsistent communication protocol of automatic monitoring equipment and limited data acquisition and transmission monitoring equipment, Guoli developed a programmable single-point multi-output intelligent data acquisition and transmission system [11].

The above descriptions of research by scholars at home and abroad have specifically mentioned big data and cloud computing. In their experiments, a large amount of data support and a large amount of calculation are required, and the process is very complicated. The experiments carried out require very professional knowledge and excellent computer technology. It is necessary to fully understand the relevant knowledge points involved. The use of machine monitoring is even more difficult to operate.

1.3. Innovations in This Article. This article focuses on solving the problem that traditional storage and processing technologies cannot meet the monitoring needs and introduces cloud computing technology and some artificial intelligence technologies to build a smart environment 
platform based on cloud computing technology. Compared with traditional environmental monitoring, this study uses cloud computing technology combined with the application of wireless sensors to monitor the environmental data of industrial clusters in real time, and through cloud computing data processing to simplify the amount of data collected by sensors while maintaining high data authenticity, reducing detected data errors. Timely positioning and tracking of abnormal data detected is of great significance to the prevention of environmental pollution.

\section{Application of Cloud Computing Technology in Intelligent Environmental Monitoring}

2.1. Cloud Computing Technology. Cloud computing technology was born under the background that the amount of Internet data storage continues to increase, and traditional database storage can no longer meet the needs of data storage. In the Internet, cloud computing is like the nerve center of the brain. It issues corresponding instructions according to the needs of users and provides users with data services and configurable computer resources. Users do not need to have in-depth exchanges with service providers and do not need to consider each kind of management work. This on-demand data interaction and service model provides users with convenient, simple, and highly operable services and is favored by more and more companies and individuals [12]. For service providers, the main features of cloud computing are distributed architecture, cloud storage technology, and virtualization technology, which are increasingly inseparable from the application of big data and artificial intelligence. Big data is the processing of massive amounts of data, which inevitably cannot be achieved through traditional database transmission and storage. It requires the help of distributed architecture of cloud computing and cloud storage and virtualization technologies. The core of artificial intelligence is to use various algorithms to predict the future through the study of large amounts of data to achieve the purpose of simulating and expanding human intelligence. This is also inseparable from the virtual storage technology and big data processing technology of cloud computing [13].

2.2. Application of Wireless Smart Sensor Technology in Environmental Monitoring. One of the main functions of the intelligent environment platform in this study is environmental quality monitoring, which requires the help of wireless intelligent sensing technology of the Internet of Things. The main monitoring content includes water quality monitoring, air quality monitoring, temperature monitoring, noise monitoring, biological monitoring, radiation monitoring, and soil quality monitoring. In order to achieve these functions, a variety of smart sensors are needed, including optics, acoustics, gases, and chemistry. Their main principles are similar. Except for the different test objects, it is based on whether a certain index of the tested substance selected at random exceeds the prescribed standard. The information is fed back to the central processing unit (CPU) of the sensor and then connected to the Internet through a wireless network interface $[14,15]$. For example, based on an electric heating temperature sensor, its principle formula is as follows:

$$
t_{T}=t_{N}+\frac{E_{T}-E_{N}}{E_{M}-E_{N}}\left(t_{M}-t_{N}\right) .
$$

Among them, $t_{T}, t_{M}$, and $t_{N}$, respectively, represent the measured temperature, the higher temperature value and the lower temperature value. $E_{T}, E_{M}$, and $E_{N}$, respectively, represent the thermoelectromotive force corresponding to these temperature values; through this formula, a more accurate actual temperature value can be obtained. A capacitive sensor is used to detect air quality. Its principle is to monitor the content of PM2.5 and other particulate matter in the air according to the different conduction rates of electrons in air and vacuum media. Its main formula is as follows:

$$
\begin{aligned}
C_{0} & =\frac{\eta_{0} \eta_{r} S}{d}=\frac{\eta_{0} \eta_{r} w h}{d}, \\
k & =\frac{\eta h}{d} \\
\eta_{0} & =8.854 * 10^{-12} \frac{\mathrm{F}}{\mathrm{m}} \\
\eta_{r} & =1 \frac{\mathrm{F}}{\mathrm{m}}
\end{aligned}
$$

where $\eta_{0}$ represents the relative permittivity in vacuum, $\eta_{r}$ represents the relative permittivity in air, $S$ represents the area of the two plates, $w, h, d$ represent the length and width of the two plates and the distance between the two plates, and $k$ is static sensitivity.

When the sensor is sampling raw data, if the raw sampling data is directly transmitted to the sensor CPU, although the obtained data can be updated in real time, it will be more realistic and reliable. However, the sampling frequency of this method is too high, and the amount of data obtained is too large, which reduces the processing efficiency of the sensor and also greatly decreases the life span of the CPU. Therefore, in smart sensors, the average value of collected sample data is often continuously updated through the smart sensor system. For example, for a set of original sampling data set $M_{0}=\left\{x_{1}, x_{2}, \ldots, x_{m}\right\}$ with a data volume of $m$, the system will take its average value as a new sampling value every $n$ times. The specific method is as follows $[16,17]$ : 


$$
\begin{aligned}
& y_{1}=\frac{1}{n} \sum_{i=1}^{n} x_{i}=\frac{x_{1}+x_{2}+\cdots+x_{n}}{n}, \\
& y_{2}=\frac{1}{n} \sum_{i=n}^{2 n} x_{i}=\frac{x_{n+1}+x_{n+2}+\cdots+x_{2 n}}{n}, \ldots y_{m / n}=\frac{1}{n} \sum_{i=m-n}^{m} x_{i}=\frac{x_{m-n+1}+x_{m-n+2}+\cdots+x_{m}}{n} .
\end{aligned}
$$

In this way, a new sampling data set $M_{1}=\left\{y_{1}, y_{2}, \ldots, y_{m / n}\right\}$ whose data volume is reduced to $m / n$ is obtained. Similarly, on this basis, the slope $k$ between the maximum value and the minimum value of the sampling data can also be used as the new sampling value.

$$
\begin{aligned}
& k_{1}=\frac{x_{m 1}-x_{n 1}}{n}, \quad\left(x_{m 1}, x_{n 1} \in\left\{x_{1}, x_{2}, \ldots . x_{n}\right\}\right), \\
& k_{2}=\frac{x_{m 2}-x_{n 2}}{n}, \quad\left(x_{m 2}, x_{n 2} \in\left\{x_{n+1}, x_{n+2}, \ldots x_{2 n}\right\}\right), \\
& \ldots \\
& k_{m / n}=\frac{x_{m n}-x_{n n}}{n}, \quad\left(x_{m n}, x_{n n} \in\left\{x_{m-n+1}, x_{m-n+2}, \ldots x_{m}\right\}\right) .
\end{aligned}
$$

This method can also obtain a new sampled data set $M_{2}=\left\{k_{1}, k_{2}, \ldots, k_{m / n}\right\}$. It should be noted that the slope between the maximum values of the sample data is used as the new sampled value. Therefore, a higher data collection frequency is required to ensure accuracy. In summary, the principle of this method is to use the properties of the mean $X$, variance $S$, and statistics $T$ to preprocess the sampled data, reduce the sampled data, and improve the processing efficiency of the sensor. Studies have shown that many phenomena in life after a large number of random experiments are statistically approximated to obey the normal distribution. This article uses the most important sample parameters $X$ and $S$ in the normal distribution to reflect the overall characteristics of the data, and it further introduces the statistic $T$ to detect abnormal values in the sample data. If $x_{i}$ in the collected data is a suspicious value, the value of the statistic $T$ is calculated. If $T$ is greater than the standard value, such data should be classified as abnormal data. This is Grubbs exception exclusion method [18].

$$
\begin{aligned}
\bar{X} & =\frac{1}{n} \sum_{i=1}^{n} x_{i}, \\
S & =\sqrt{\frac{1}{n} \sum_{i=1}^{n}\left(X_{i}-\bar{X}\right)^{2},} \\
T & =\frac{X_{i}-\bar{X}}{S}, \quad(i=1,2, \ldots, n) .
\end{aligned}
$$

2.3. Intelligent Recognition Algorithm in Image Processing. In the intelligent environment platform, the collected images and video data need to be processed and restored through certain technologies. Among them, the most common interference is the influence of light and color, so whether it is sensors, various monitoring, and equipment with shooting functions, all need intelligent recognition technology to assist image processing [19] intelligent recognition technology. This article mainly introduces the processing technology of the light part of the image in the intelligent recognition technology. The traditional color system (RGB) color space model consists of three color components: red, green, and blue, but it is found that the mutual influence of the three colors is not conducive to color adjustment. Therefore, on this basis, this article introduces the HSI color space that is more in line with people's perception of external color habits and the $\mathrm{YCbCr}$ color model is commonly used in various video playback screen systems [20]. The principle formula for converting RGB color space to color space is as follows:

$$
\begin{aligned}
r & =\frac{R}{R+G+B}, \\
g & =\frac{G}{R+G+B}, \\
b & =\frac{B}{R+G+B}, \\
H & =\arccos \left[\frac{(r-g)+(r-b)}{2 \sqrt{(r-g)^{2}+(r-b)(g-b)}}\right] \\
S & =1-\frac{3 \min (r, g, b)}{r+g+b}, \\
I & =\frac{r+g+b}{3} .
\end{aligned}
$$

In image intelligent recognition, there are many commonly used detection algorithms. This article mainly introduces the AdaBoost algorithm, which is widely used in face recognition and also plays an important role in this research. Its main principle is to allow the computer to continuously classify and learn data features through algorithms. For example, a simple application example of intelligent recognition of human faces and non-human faces in images. If a set of image data is $\left\{\left(x_{i}, y_{i}\right) \mid i=1, \ldots, n\right\}$, use $y=1$ and $y=0$ to represent the collected sample image data as human face and non-human face. After $N$ learning and classification, according to whether the image is a face data information, use $y$ and $n$, respectively, to represent the number of samples with human faces and non-human faces. Then, the weight of the sample data can be obtained [21]. 


$$
\begin{aligned}
\varphi_{1, j} & =\frac{1}{2 y}, \frac{1}{2 n}, \\
m_{i, j} & =\frac{\varphi_{i, j}}{\sum_{j=1}^{n} \varphi_{i, j}}, \quad i=1, \ldots, N .
\end{aligned}
$$

According to the mapping relationship between the single characteristic $p$ of the sample and the weak separator $f$, all samples can be continuously classified by the association rule, and the error classification rate $\mu$ can be obtained based on this. The calculation formula is as follows:

$$
\mu_{p}=\sum_{i=1}^{n} \varphi_{i}\left|f\left(x_{i}, p, q, \lambda\right)-y_{i}\right| .
$$

In each calculation process, the classifier that minimizes the error classification rate is selected for the next round of classification. This classifier is the best weak classifier $f_{l}$. After $N$ times of learning, $N$ best weak classifiers can be obtained. A strong classifier can be obtained by weight calculation and combination, where $\lambda_{l}$ is the parameter $\lambda$ value when the classifier is selected as the best weak classifier.

$$
F(x)=\left\{\begin{array}{l}
1, \sum_{i=1}^{N} \lambda_{l} f_{l}\left(x_{i}\right) \geq \frac{1}{2} \sum_{i=1}^{N} \lambda_{i}, \\
0, \text { otherwise. }
\end{array}\right.
$$

2.4. Location and Tracking Algorithm in Intelligent Environment. In the intelligent environment detection platform, for the abnormal data detected, it is sometimes necessary to carry out location tracking investigation. This is widely used in police systems, but it is also widely used in other areas [22]. Generally speaking, target location and tracking methods are realized by establishing models. For example, take the wireless sensor's target positioning and tracking of the detection data as an example. In the coordinate system, the sensor is used as the origin of the coordinate to monitor the moving position of the target. If the target moves in a straight line in the area, a positioning algorithm based on RSSI ranging can be used, which can establish a gradual model of signal strength and distance [23].

$$
y(d)=y\left(d_{0}\right)-10 m \log \left(\frac{d}{d_{0}}\right) .
$$

Among them, $d_{0}$ is usually set to 1 meter, which means the reference distance, $d$ means the distance between the sensor transmitting the signal and the receiving signal terminal, $y(d), y\left(d_{0}\right)$ are the signal strength when the distance between the sensor sending signal and the receiving signal position is $d, d_{0}$, generally expressed by power, $m$ is the weakening index from the signal sending to the receiving process. The longer the distance, the weaker the signal and the larger the value of $m$. If the target is moving in a uniform straight line, the target tracking and simulation motion model can be established according to the principle of unscented Kalman filter [24].

$$
\begin{aligned}
& Y(m)=\varepsilon Y(m-1)+\Gamma u(m) \\
& Z(m)=\sqrt{\left(x_{m}-x_{0}\right)^{2}+\left(y_{m}-y_{0}\right)^{2}}+v(m) .
\end{aligned}
$$

2.5. Least Squares Method in Data Processing. In the intelligent environment platform of industrial clusters, in order to reduce the error caused by the real-time monitoring of changes in environmental factors in various industrial areas, the environmental data values obtained by the detection should be more authentic and reliable. This requires the use of scientific data processing methods to process the collected data after detecting and uploading environmental data through smart sensors. The commonly used method is the least squares method. For example, for the temperature and humidity of the industrial area, the distribution of PM2.5 in the air, and other environmental values that change over time, after collecting the original data, the number of samples can be reduced by the abovementioned methods, and then the algorithm can be used to upload these. The data is processed twice. The principle of the least squares method is to curve-fit the collected data. The idea of regression is also used here. Compared with the original data, the processed data is approximately distributed on a curve, which is generally called this. The curve is a fitting curve, sometimes called a regression curve. The data curve obtained in this way is smoother, and some abnormal points collected due to some factors during the detection can be removed, the experimental error is reduced, and the data obtained is closer to the true value $[25,26]$.

Using the least squares method to process data can analyze and predict environmental data more intuitively and clearly. When the least squares method is used to curve-fit sampled data, the equation of the fitted curve cannot be directly obtained, so the idea of regression is required. First, find the equation of the regression line of these data. For example, suppose a set of sampled data is an array set $\left\{\left(x_{i}, y_{i}\right) \mid i=1, \ldots, n\right\}$ with a data volume of $n$, and the data points in these arrays are presented through coordinates, and it is found that they are all distributed near a straight line. This line is a regression line or a fitted line. The equation is as follows [27]:

$$
Y_{m}=k x+b, \quad k, b \in R .
$$

The determination of this linear equation only requires the values of $k$ and $b$, and the actual detected value and the calculated value are processed by the least squares method to obtain new sampling data. This is a data simplification process.

$$
\omega=\sum\left(Y_{l}-Y_{m}\right)^{2}=\sum\left(Y_{l}-k X_{l}-b\right)^{2} .
$$

When $\omega$ is the smallest, use the above formula to find the partial derivatives of $k$ and $b$, respectively, and make the partial derivatives zero; then, the equation for solving $k$ and $b$ can be obtained as follows: 


$$
\begin{aligned}
& k=\frac{\sum X_{l} Y_{l}-\sum X_{l} \sum Y_{l}}{\sum X_{l}^{2}-\left(\sum X_{l}\right)^{2}}, \\
& b=\sum Y_{l}-k \sum X_{l} .
\end{aligned}
$$

Substituting $k$ and $b$ into (10), we can obtain the regression line equation, and then further find the fitting curve equation through the line equation. The process of finding the fitting curve equation is actually to find the curve equation $y=F(x)$ when the sum of the squares of the distance from the point on the sampled data set $\left\{\left(x_{i}, y_{i}\right) \mid i=1, \ldots, n\right\}$ is the smallest. $F(x)$ can be obtained by minimizing the sum of squares of the error. The formula for the sum of squares of error is as follows:

$$
e^{2}=\sum\left[F\left(X_{i}\right)-Y_{i}\right]^{2} .
$$

\section{Experimental Research on Cloud Computing Technology in Environmental Intelligence}

3.1. Research Object. The research object of this paper is the design of intelligent environment platform based on cloud computing in industrial clusters. In the era of cloud computing, information collection and sharing in various industries are gradually moving closer to cloud computing, and environmental monitoring in industrial clusters is also inseparable from the application of cloud computing. Based on the difficulty of data collection in the environmental monitoring of the current industrial clusters, the large scale of sampled data, and the large amount of data, traditional storage, and processing technologies cannot meet the needs of real-time monitoring and updating of environmental data, research the introduction of cloud computing technology and some artificial intelligence technologies to build industrial clusters. The district intelligent environment platform provides more intelligent and real-time environmental data information services for enterprises, individuals, and environmental supervision departments in industrial clusters so that enterprises and individuals can pay attention to environmental changes and protect them from pollution and supervision departments for environmental supervision and governance.

3.2. Experimental Design. This study collects data on environmental supervision in industrial clusters by searching for relevant documents, online surveys, and questionnaire surveys, and combining some related research results to design a cloud computing-based intelligent environment platform for industrial clusters. The experiment is divided into four steps: one is to collect data and organize data, and conduct demand analysis and technical understanding of the intelligent environment platform. The second is to determine the functions that the platform needs to implement based on demand analysis and use UML use case diagrams and overall structure diagrams to determine the functional modules of the platform. The third is to use cloud computing virtual storage and distributed data processing technologies to realize the functions of the intelligent environment platform, which uses the image intelligent recognition algorithm, the target positioning algorithm based on RSSI ranging, and the unscented Boolean man tracking algorithm to assist common cloud computing algorithms with achieving these functions. Fourth, the least squares method performs curve fitting on the collected environmental data to predict the environmental conditions and changing trends in the sampling area and puts forward some suggestions on the construction of intelligent environmental platforms and the prevention and treatment of environmental pollution. In the process of building the platform, due to insufficient understanding and application of cloud computing technology, some technical problems were encountered, and solutions were found by consulting relevant documents and consulting professionals. For example, the amount of original sampling data obtained by wireless sensor sampling is large, and some data have measurement errors, which are not conducive to analysis. Therefore, in the process of processing these data by the platform, some algorithms are used to transform the data, reduce the range of sampled data, and retain high accuracy.

\subsection{Experimental Method}

3.3.1. Literature Research Method. In the preparatory work of this study, firstly, through the academic research database resources such as HowNet, Wanfang, Duxiu, and Bailian, the relevant research literature was consulted to have a full understanding of the status quo of environmental monitoring in industrial clusters and some research results. Analyze the necessity of building a smart environment platform based on cloud computing based on the hazards and losses caused by environmental pollution in industrial clusters, and determine the functional requirements of the smart environment platform, including environmental monitoring, location tracking, environmental information sharing, and environmental pollution prediction.

3.3.2. Internet Investigation Method. This paper conducts a network survey on the environmental concerns of enterprises in various industrial agglomeration areas by visiting the public platform information of enterprises in various industrial agglomeration areas. Analyzing the company's attention to various environmental data indicators and the composition of the company's environmental management, it is found that some companies only pay attention to the environmental quality standards in their own industrial areas and do not pay attention to the environmental conditions of other areas in the region. Combined with the field visit, it was found that this was caused by inaccurate environmental monitoring data in some areas and the environment in some areas was not included in the monitoring scope. Secondly, some environmental monitoring sensor equipment is relatively backward, the environmental data information processing efficiency is low, and the environmental indicators cannot be monitored in real time, and the detected data errors are 
TABLE 1: Ways for users in industrial clusters to obtain environmental information.

\begin{tabular}{|c|c|c|c|c|c|c|c|}
\hline & \multirow{2}{*}{$\begin{array}{l}\text { Follow the } \\
\text { crowd }\end{array}$} & \multicolumn{2}{|c|}{$\begin{array}{l}\text { Government, } \\
\text { online media }\end{array}$} & \multicolumn{2}{|c|}{$\begin{array}{c}\text { Environment } \\
\text { protection department }\end{array}$} & \multicolumn{2}{|c|}{$\begin{array}{c}\text { Corporate environmental } \\
\text { monitoring }\end{array}$} \\
\hline & & Number & Proportion (\%) & Number & Proportion (\%) & Number & Proportion (\%) \\
\hline \multirow{3}{*}{ Personnel } & Enterprise & 71 & 44.3 & 69 & 43.1 & 69 & 43.1 \\
\hline & Employee & 66 & 41.2 & 56 & 35 & 56 & 35 \\
\hline & $\begin{array}{c}\text { Environmental } \\
\text { protection department }\end{array}$ & 46 & 28.8 & 47 & 29.4 & 47 & 29.4 \\
\hline \multirow{3}{*}{ Age } & $16-35$ & 68 & 42.5 & 63 & 39.4 & 63 & 39.4 \\
\hline & $36-55$ & 53 & 33.1 & 55 & 34.4 & 55 & 34.4 \\
\hline & $56-80$ & 51 & 31.9 & 67 & 41.9 & 67 & 41.9 \\
\hline
\end{tabular}

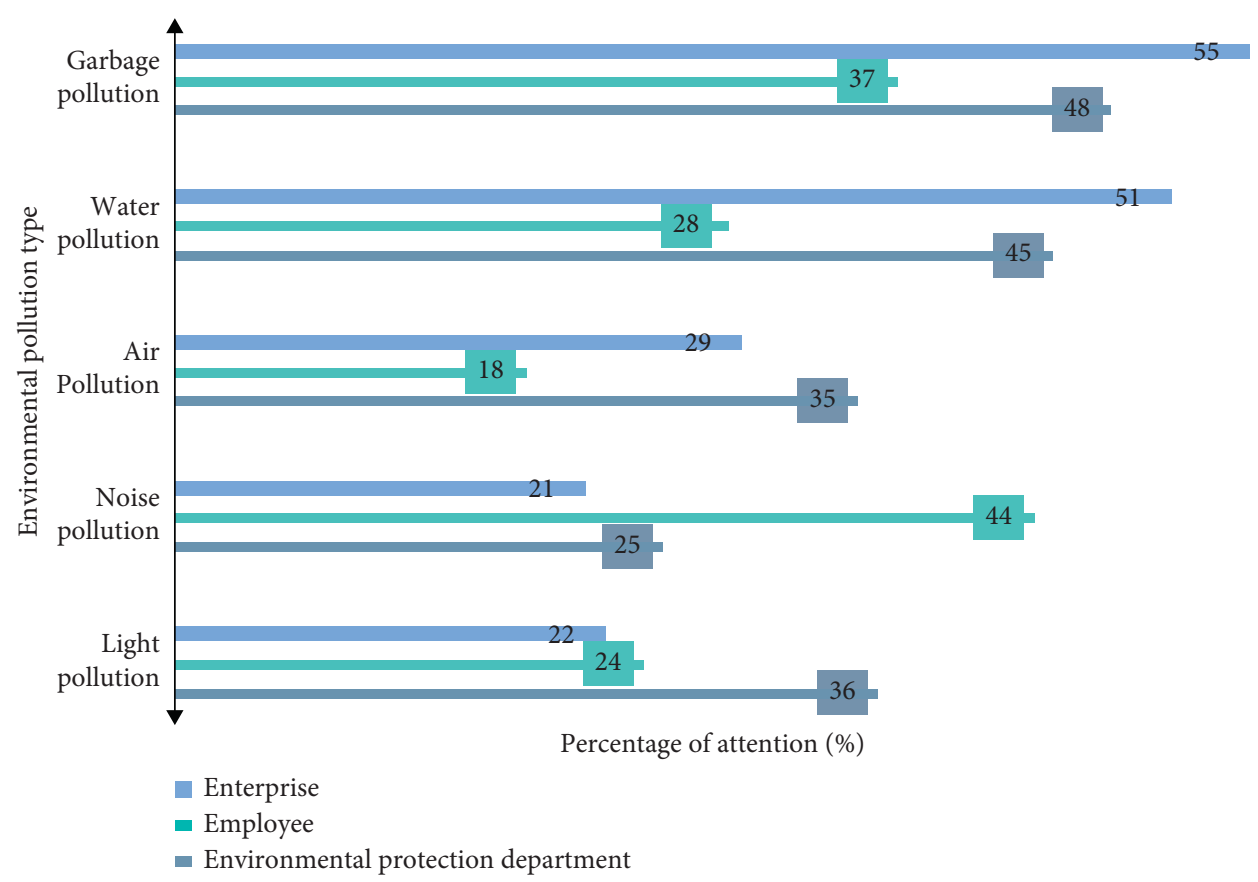

FIgURE 1: People's attention to various environmental pollution information.

large. Based on these references, this research proposes corresponding improvement measures for these problems.

\subsubsection{Questionnaire Survey Method. A questionnaire survey} was conducted on the residents in the industrial agglomeration area, which are mainly corporate employees, to understand some of the employees' opinions and suggestions on environmental supervision and governance in the industrial agglomeration area and to find out the shortcomings of the current environmental supervision in the industrial agglomeration area.

\section{Discussion}

4.1. Demand Analysis of Intelligent Environment Platform in Industrial Clusters. In order to investigate the environmental monitoring of industrial clusters in depth, the relevant personnel of this study conducted field visits and questionnaire surveys on the people in the industrial clusters. From the survey results in Table 1, it is found that there are three main ways for enterprises and individuals in industrial clusters to obtain environmental information. Among them, the largest proportion is environmental information released by the government and various online media news, with a maximum proportion of $44.3 \%$, followed by the application to the local environmental protection department to consult relevant environmental information, and finally the environmental monitoring data and personal observation results of the company's voluntary organization.

According to the above survey results, we can also find that in industrial clusters, both companies and individuals rarely pay attention to environmental information through the environmental protection department and even do not pay much attention to the environment. This stems from the fact that people's environmental awareness is still not strong enough, and the way to obtain environmental information is single, and the accuracy and credibility are too low. As 


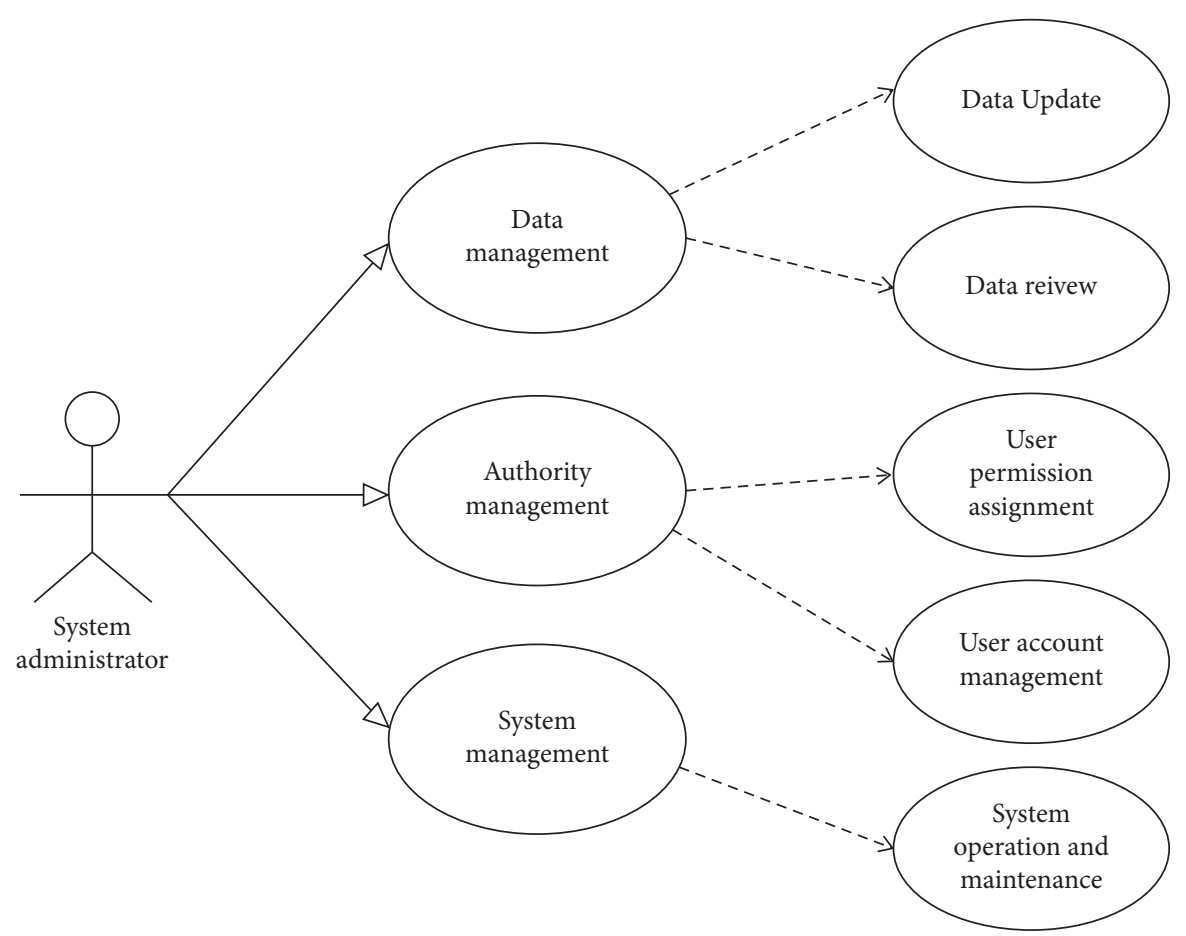

FIgURE 2: System administrator UML use case diagram.

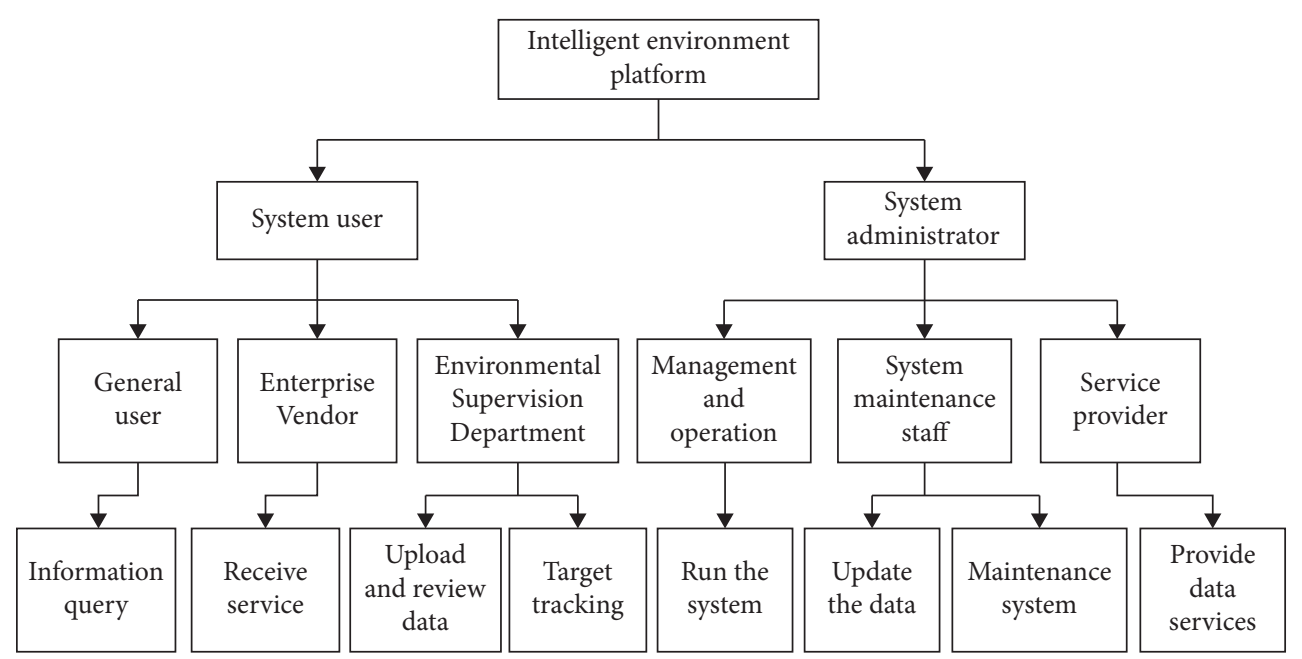

FIGURE 3: Organization chart of intelligent environment platform.

shown in Figure 1, people pay more attention to visible environmental issues, such as garbage pollution, sewage discharge, and PM2.5, but pay less attention to some potential environmental pollution hazards.

\subsection{Design of Intelligent Environment Platform in Industrial} Cluster. According to the demand analysis of the intelligent environment platform, this paper studies and designs an intelligent environment platform based on cloud computing technology. Figure 2 shows the use case diagram of the platform system administrator. It mainly displays the authority and service functions of the system administrator, mainly including data management, account information management, and system management. The specific work content is real-time update, upload, and review of data, as well as management of user account permissions and other information and system background operation and maintenance.

After clarifying the permissions of administrators and ordinary users and the corresponding service types, this article introduces the main functional modules of the platform through the overall structure of the platform system. As shown in Figure 3, according to the different permissions of different users, the platform will allocate different service interfaces after entering the main interface, 


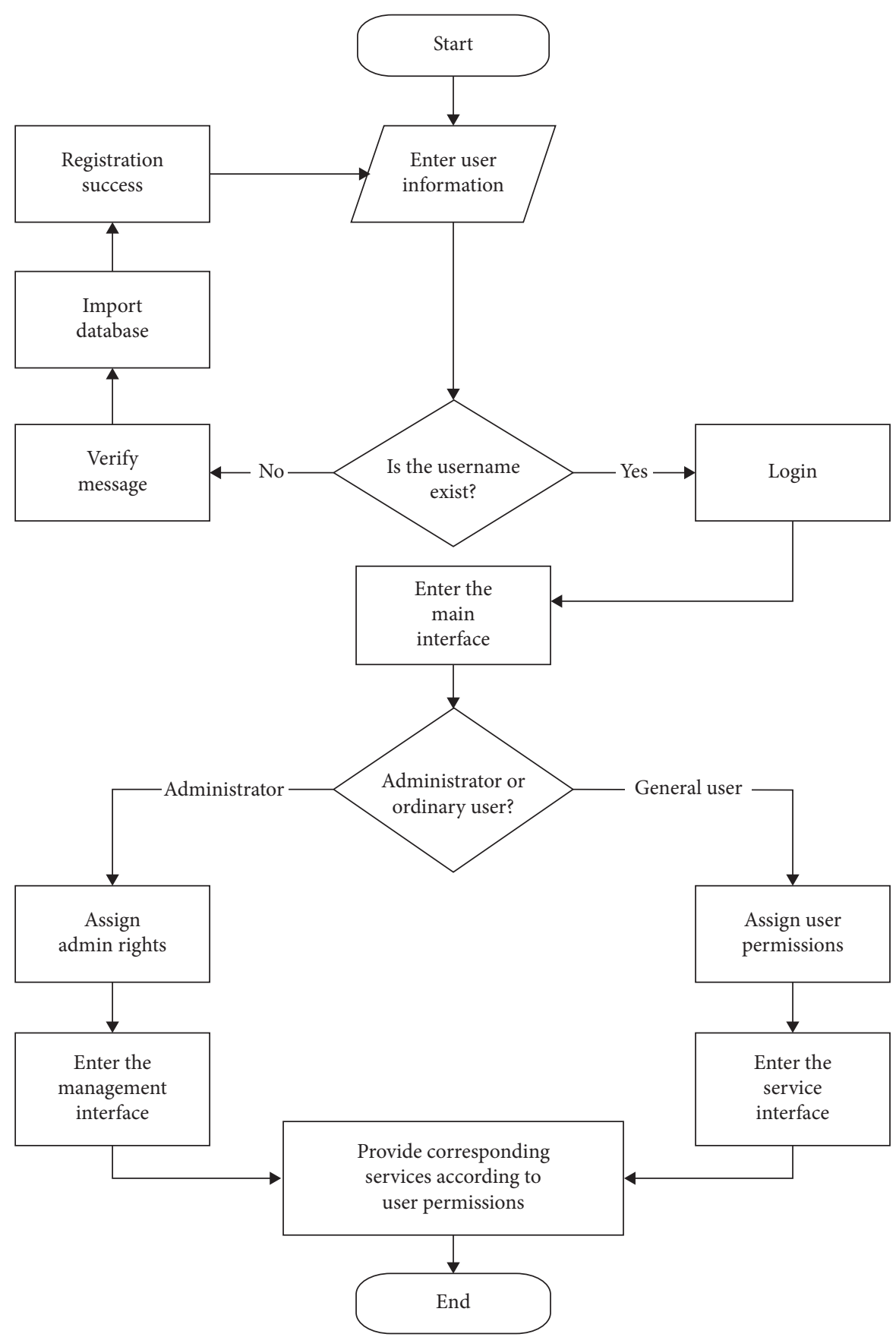

FIGURE 4: Intelligent environment platform system flow chart.

and users can obtain corresponding environmental information and other services.

Figure 4 is the system flow chart of the operation of the smart environment platform. The user first enters the account number, password, and other corresponding information in the registration/login interface, and the system determines whether there is corresponding account information in the database. If not, enter the registration interface and enter the relevant information. After being reviewed by the administrator, it is entered into the database, and the user can log in, and then obtain the corresponding environmental information and other services according to different permissions.

4.3. Analysis of Temperature and Humidity Monitoring and Prediction Accuracy in Industrial Clusters. The main functions of this smart environment platform are environmental monitoring, environmental pollution prevention alarms, etc. The following takes the temperature and humidity monitoring in one of the low-temperature industrial areas as an example to 
TABLE 2: Temperature and humidity monitoring and prediction accuracy.

\begin{tabular}{|c|c|c|c|c|c|c|}
\hline & \multicolumn{3}{|c|}{ Temperature monitoring } & \multicolumn{3}{|c|}{ Humidity monitoring } \\
\hline & Raw data $\left({ }^{\circ} \mathrm{C}\right)$ & Processed data $\left({ }^{\circ} \mathrm{C}\right)$ & Prediction accuracy (\%) & Raw data (\%) & Processed data (\%) & Prediction accuracy (\%) \\
\hline 0 & 16 & 15 & 89.7 & 90.8 & 91.4 & 78.6 \\
\hline 2 & 13 & 15.5 & 91.6 & 92.5 & 91.8 & 85.2 \\
\hline 4 & 15 & 16 & 88.7 & 92.7 & 92.1 & 86.9 \\
\hline 6 & 19 & 17 & 92.2 & 94 & 92.5 & 78.8 \\
\hline 8 & 19.5 & 18 & 93.8 & 92.6 & 93.3 & 89.6 \\
\hline 10 & 17 & 19 & 90.5 & 93.7 & 94.5 & 90.5 \\
\hline 12 & 18 & 20 & 87.6 & 97.5 & 96.5 & 93.8 \\
\hline 14 & 21 & 19.7 & 89.8 & 98 & 97.5 & 92.4 \\
\hline 16 & 19 & 18 & 91.2 & 97.5 & 96.5 & 90.5 \\
\hline 18 & 17 & 15.5 & 85.4 & 94.3 & 95 & 88.3 \\
\hline 20 & 14 & 15 & 77.9 & 93.5 & 94.2 & 91.6 \\
\hline 22 & 13 & 16 & 93.2 & 96 & 95 & 89.9 \\
\hline
\end{tabular}

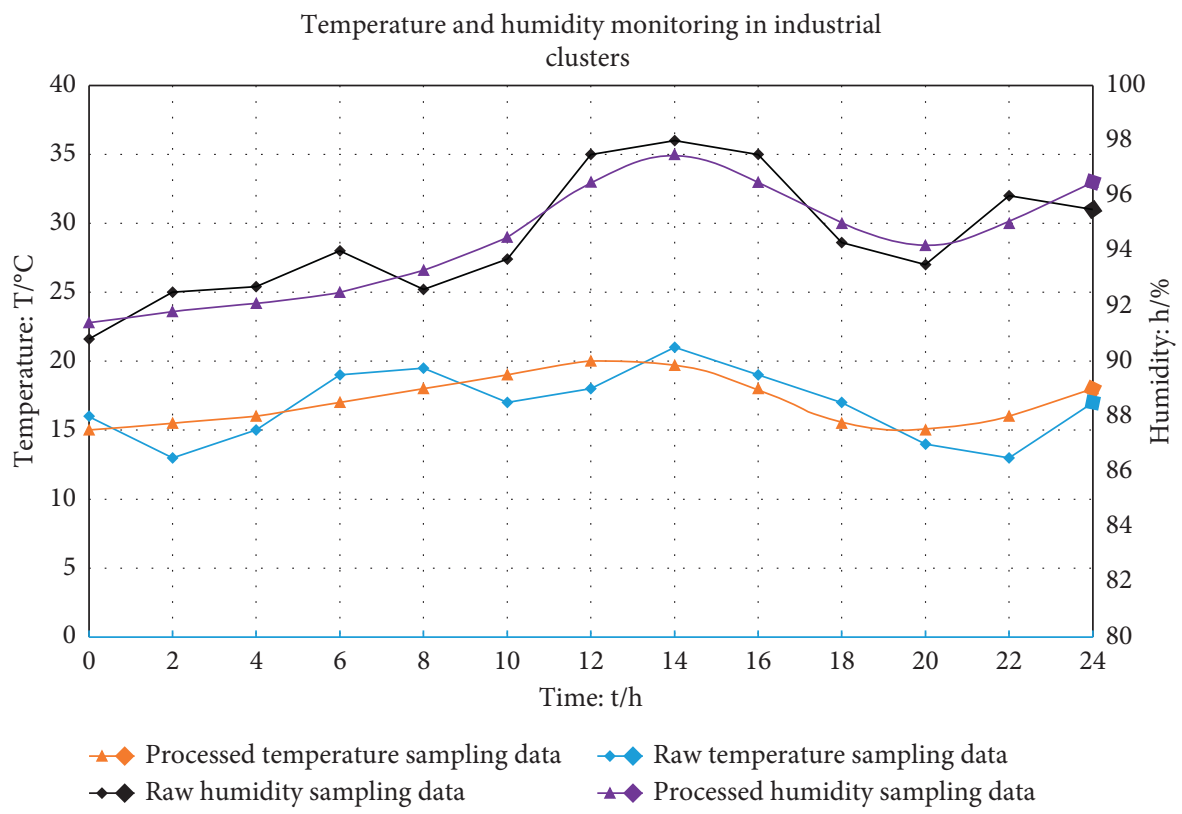

FIGURE 5: Temperature and humidity monitoring in industrial clusters.

analyze the temperature and humidity changes in the industrial area and predict the temperature in the future. The humidity change trend is shown in Table 2.

Based on the monitoring data collected above and the data processed by cloud computing, the linear graph of the temperature and humidity change trend in the industrial area is drawn as shown in Figure 5 below. It can be found that the sampled raw data fluctuates near the fitting curve obtained after data processing, but the general trend is in line. Moreover, comparing the prediction data, it is found that this curve fitting method has a higher prediction accuracy for temperature and humidity changes.

\section{Conclusions}

This article first conducts certain investigations on the environmental conditions of industrial clusters, analyzes and summarizes some of the problems existing in the environmental testing of industrial clusters, and understands the current safety issues and deficiencies in the environmental management of industrial clusters. In view of these problems, the functions that the intelligent environment platform should have are proposed. Based on the diverse types of sensor data collection, complex conditions, and large amount of data, it is proposed to use cloud computing technology to build an intelligent environmental platform to assist in the prevention and control of environmental pollution. Through experiments, the application effect of the intelligent environment platform is studied, compared with related environmental monitoring and management research at home and abroad, the defects of the existing environmental monitoring technology are found, and the research plan of the intelligent environment platform is demonstrated and analyzed.

Through case analysis and experimental demonstration, the cloud computing technology-based intelligent environment platform for industrial clusters designed in this study has the following innovations. Firstly, the combination 
of wireless sensors and the Internet can enable real-time updates of monitored environmental data, so sampling the data obtained is more realistic and concrete. Secondly, the introduction of cloud computing technology can solve the problem of insufficient storage space for a large amount of environmental data generated by real-time monitoring and inconvenience of analysis and processing. This depends on the characteristics of cloud computing storage virtualization and distributed architecture and the mode of providing services on demand. Then, in the process of data processing, the least squares method and various cloud-based algorithms are used to process the data after certain improvements and optimizations. Not only can we get more concise and intuitive data, but also more accurate description and predict the environmental conditions in the sampling area. Finally, the use of several positioning and tracking algorithms can monitor the environmental conditions at any location in the industrial agglomeration area at any time, and positioning and tracking play an important role in the prevention and warning of environmental pollution and some hidden safety hazards. For example, air, temperature, and thermal energy sensors can quickly locate and track the location of a fire, minimizing the damage and loss of the disaster. In the future, the application of cloud computing in intelligent environments can continue to be optimized and deepened.

\section{Data Availability}

No data were used to support this study.

\section{Conflicts of Interest}

The author declares that there are no conflicts of interest with any financial organizations regarding the material reported in this manuscript.

\section{Acknowledgments}

This work was supported by the Major Project of Beijing Social Science Foundation "Research on Financial Support System Adapting to the Coordinated Development of Strategic Emerging Industries in Beijing-Tianjin-Hebei” (no. 20ZDA11).

\section{References}

[1] W. Wu, Y. Liu, C.-H. Wu, and S.-B. Tsai, “An empirical study on government direct environmental regulation and heterogeneous innovation investment," Journal of Cleaner Production, vol. 254, Article ID 120079, 2020.

[2] S. K. Biswas, D. Devi, and M. Chakraborty, "A hybrid case based reasoning model for classification in Internet of things (iot) environment," Journal of Organizational and End User Computing, vol. 30, no. 4, pp. 104-122, 2018.

[3] C. T. Yang, S. T. Chen, and W. Den, "Implementation of an intelligent indoor environmental monitoring and management system in cloud," Future Generation Computer Systems, vol. 96, no. 9, pp. 731-749, 2018.

[4] J. Zhang, Z. Chen, Z. Xu, M. Du, W. Yang, and L. Guo, “A distributed collaborative urban traffic big data system based on cloud computing," IEEE Intelligent Transportation Systems Magazine, vol. 11, no. 4, pp. 37-47, 2019.

[5] J. Wan, M. Yi, and D. Li, "Mobile services for customization manufacturing systems: an example of industry 4.0," IEEE Access, vol. 54, no. 6, p. 1, 2016.

[6] T. Zhao, J. Tang, and X. Chen, "A data acquisition system in intelligent environmental monitoring device for industrial field," IOP Conference Series: Earth and Environmental Science, vol. 170, no. 3, Article ID 032045, 2018.

[7] S. A. Ahmed, V. L. Popov, and A. V. Topalov, "Environmental monitoring using a robotized wireless sensor network," $A I$ \& Society, vol. 33, no. 2, pp. 1-8, 2018.

[8] V. Scilimati, A. Petitti, P. Boccadoro et al., "Industrial Internet of things at work: a case study analysis in robotic-aided environmental monitoring," IET Wireless Sensor Systems, vol. 7, no. 5, pp. 155-162, 2017.

[9] S. Laghari and M. A. Niazi, "Modeling the Internet of things, self-organizing and other complex adaptive communication networks: a cognitive agent-based computing approach," PLoS One, vol. 11, no. 1, Article ID e0146760, 2016.

[10] N. K. Pareek and V. Patidar, "Medical image protection using genetic algorithm operations," Soft Computing, vol. 20, no. 2, pp. 763-772, 2016.

[11] Q. U. Guoli, W. U. Yun, and X. U. Gaihua, "Embedded programmable single point multiple output intelligent data acquisition and transmission system," Asian Agricultural Research, vol. 11, pp. 58-64, 2016.

[12] S. Singh and I. Chana, "Cloud resource provisioning: survey, status and future research directions," Knowledge and Information Systems, vol. 49, no. 3, pp. 1005-1069, 2016.

[13] P. Lou, S. Liu, J. Hu, R. Li, Z. Xiao, and J. Yan, "Intelligent machine tool based on edge-cloud collaboration," IEEE Access, vol. 8, no. 11, pp. 139953-139965, 2020.

[14] H. Kang, M. Kim, M. Bae, H.-C. Bang, and H. Yoe, "A conceptual device-rank based resource sharing and collaboration of smart things," Multimedia Tools and Applications, vol. 75, no. 22, pp. 14569-14581, 2016.

[15] C.-W. Jeong, S. G. Lee, J. Lee, K.-H. Yoon, and S. C. Joo, "Construction of dynamic medical information system for digital hospital environments," Wireless Personal Communications, vol. 91, no. 4, pp. 1575-1590, 2016.

[16] J. Niu, X. Li, J. Gao, and Y. Han, "Blockchain-based anti-keyleakage key aggregation searchable encryption for IoT," IEEE Internet of Things Journal, vol. 7, no. 2, pp. 1502-1518, 2020.

[17] T. Qiu, Z. Zhao, T. Zhang, C. Chen, and C. L. P. Chen, "Underwater Internet of things in smart ocean: system Architecture and open issues," IEEE Transactions on Industrial Informatics, vol. 16, no. 7, pp. 4297-4307, 2020.

[18] H. Tang, D. Li, J. Wan, M. Imran, and M. Shoaib, “A reconfigurable method for intelligent manufacturing based on industrial cloud and edge intelligence," IEEE Internet of Things Journal, vol. 7, no. 5, pp. 4248-4259, 2020.

[19] A. Ghani, H. A. Naqvi, M. Sher, Z. S. Khan, I. Khan, and M. Saqlain, "Energy efficient communication in body area networks using collaborative communication in Rayleigh fading channel," Telecommunication Systems, vol. 63, no. 3, pp. 357-370, 2016.

[20] S.-S. Han, Y.-K. Kim, Y.-B. Jeon et al., "Distributed deep learning platform for pedestrian detection on IT convergence environment," The Journal of Supercomputing, vol. 76, no. 7, pp. 5460-5485, 2020.

[21] Y. Lu, L. Liu, J. Panneerselvam, B. Yuan, J. Gu, and N. Antonopoulos, "A GRU-based prediction framework for intelligent resource management at cloud data centres in the 
age of 5G," IEEE Transactions on Cognitive Communications and Networking, vol. 6, no. 2, pp. 486-498, 2020.

[22] Z. Li, W. Tarneberg, and M. Kihl, "Using a predator-prey model to explain variations of cloud spot price," Journal of Intelligent and Fuzzy Systems, vol. 28, no. 6, pp. 2679-2689, 2017.

[23] M. S. M. Dhar and R. Manimegalai, "A policy-oriented secured service for the e-commerce applications in cloud," Personal and Ubiquitous Computing, vol. 22, no. 5-6, pp. 911-919, 2018.

[24] G. Norris, "Rolls-royce doubles down on digital strategy for engines," Aviation Week \& Space Technology, vol. 180, no. 4, p. 35,2018 .

[25] R. Nan and H. Zhang, "Multimedia learning platform development and implementation based on cloud environment," Multimedia Tools and Applications, vol. 78, no. 24, pp. 35651-35664, 2019.

[26] X. Tang, H. Xu, and Y. Tan, "Personalized recommendation mechanism based on collaborative filtering in cloud computing environment," International Journal of Information Technology and Web Engineering, vol. 12, no. 3, pp. 11-27, 2018.

[27] C.-C. Lin, D.-J. Deng, and C.-C. Yao, "Resource allocation in vehicular cloud computing systems with heterogeneous vehicles and roadside units," IEEE Internet of Things Journal, vol. 5, no. 5, pp. 3692-3700, 2018. 